\title{
El enfoque de las capacidades y las competencias transversales en el EEES
}

\author{
Gloria Alarcón García \\ Universidad de Murcia, España \\ gloria@um.es \\ Cristina GuIRAo Mirón \\ Universidad de Murcia, España \\ cguirao@um.es
}

\begin{abstract}
Resumen
Tras una breve exposición de la teoría del enfoque de las capacidades de Amartya Sen y de Martha C. Nussbaum se relaciona esta teoría con las competencias transversales fijadas por el EEES. El objetivo de este trabajo es el de analizar si las capacidades fundamentales han sido recogidas o no en las competencias transversales del EEES y en qué medida y cuáles de ellas se desarrollan en mayor proporción. Para ello se ha realizado un estudio experimental con estudiantes de último grado de la Facultad de Economía.
\end{abstract}

Palabras clave: EEES, teoría del enfoque de las capacidades, competencias transversales, Martha Nussbaum.

The capabilities approach and transversal skills: the case of economic studies

\begin{abstract}
In this article, after a summary of the theory of capabilities approach of Amartya Sen and Martha C. Nussbaum, we relate this theory to generic skills set by the EEES. Our goal is to analyze whether the fundamental capabilities have been included, or not, in the EEES generic skills, and to the extent and which of them have been developed. In order to carry out this analysis, we conducted a survey with students from the Faculty of Economics at the University of Murcia.
\end{abstract}

Key Words: EEES, the capabilities approach, transferable skills, Martha Nussbaum.

Referencia normalizada:

Alarcón García, G. y Guirao Mirón, C. (2013) El enfoque de las capacidades y las competencias transversales en el EEES. Historia y Comunicación Social. Vol. 18. № Especial Diciembre. Págs. 145-157.

Sumario: 1. Introducción 2. Metodología 3. Marco teórico 3.1. El enfoque de las capacidades como marco teórico del desarrollo de competencias educativas 3.2. Las competencias transversales del EEES y su relación con las capacidades 4. Resultados y discusión 5. Conclusiones 6 . Referencias bibliográficas 7. Notas 8. Anexo. 


\section{Introducción}

El concepto de educación implícito en la teoría del enfoque de las capacidades vincula ésta al desarrollo socioeconómico de los países. En 1998, Amarthya Sen, premio nobel de economía, insistía en la importancia de la educación, no sólo como un agente determinante del crecimiento y desarrollo económico de los países, sino también, del desarrollo y del bienestar de las personas.

El argumento no era novedoso puesto que ya las teorías del capital humano (Solow, 1956; Schultz, 1961; Becker, 1964), teorizaban sobre la importancia de la educación en el desarrollo económico de los países, sosteniendo que la educación, en el sentido de la inversión en capital humano, es uno de los factores determinantes de una mayor productividad. Igual que el capital físico, el capital humano, "entendido como un stock de conocimientos, capacidades y habilidades que potencian la productividad del ser humano, pueden ser considerados como factores de producción en la medida en que ambos pueden ser acumulados" (Briceño, 2011: 49). Por tanto, en esta teoría, la educación se convierte en el principal elemento de formación de capital humano, factor fundamental para el aumento de la productividad y el desarrollo.

Hasta ahora, los estudios realizados sobre desarrollo de capacidades y EEES (Melanie Walker, 2006) han elaborado listas de capacidades, que el paso por la educación superior debería desarrollar en los alumnos. En nuestro artículo, partiendo de una breve exposición de la teoría del enfoque de las capacidades de Amartya Sen y de Martha C. Nussbaum, así como del listado de las competencias transversales fijadas por el EEES, pondremos en conexión las unas con las otras, con la pretensión de analizar si todas las capacidades han sido recogidas o no en las competencias transversales, en qué medida y cuáles de ellas se desarrollan en mayor proporción. En el apartado siguiente expondremos la metodología utilizada en un estudio experimental con estudiantes y al final del artículo presentaremos los primeros resultados y las conclusiones, tras un sencillo análisis estadístico, de cómo se han desarrollado las capacidades educativas a partir de las competencias transversales. Por último, realizaremos algunas propuestas para el diseño de las competencias, en aquellos casos en los que no se haya tenido en cuenta alguna de las capacidades, que consideramos imprescindible de desarrollar en el EEES.

\section{Metodología}

Siendo el objeto de nuestra investigación, conocer si los alumnos y alumnas, adquiriendo las competencias transversales recogidas en los currículums de grado, han desarrollado las capacidades básicas que les permita su bienestar, elaboramos un cuestionario en el que pusimos en relación la lista de capacidades elaborada por Melanie Walker (2006), con las competencias transversales. Esto es, seleccionamos y relacionamos las capacidades con las diferentes competencias transversales 
del EEES, distinguiendo entre instrumentales, interpersonales y sistémicas y, como resultado de ello, elaboramos un cuestionario que fue cumplimentado en la primera quincena de septiembre de 2013.

Así, el cuestionario se conformó por 40 ítems, el cual fue respondido por el alumnado de $4^{\circ}$ curso del Grado en Economía de la Facultad de Ciencias Económicas y Empresariales de la Universidad de Murcia. Propiamente, el ejercicio consistió en que cada alumno y alumna tenía que evaluar en una escala Likert del 1 al 10 en qué medida consideraba que sus estudios universitarios, o su paso por la Universidad, le habían dotado o desarrollado las capacidades, siendo el 10 la puntuación dada a la más relevante -la que más desarrollaba cada una de esas capacidades- y 1 , la menos relevante. De él, por tanto, se pretendía extraer cuál había sido su experiencia y su valoración en la adquisición de capacidades a través de las competencias transversales del grado en Economía.

La lista de capacidades utilizada en este trabajo se corresponde con la realizada por M. Walker (2006) para el EEES. Hemos añadido, el "empoderamiento", pues consideramos una capacidad que la educación superior debería desarrollar en alumnas y alumnos, para el logro de una sociedad más igualitaria y más constructiva .De este modo la lista de capacidades básicas utilizada por nosotras en el experimento es la siguiente:

1. Razón práctica

2. Resiliencia

3. Conocimiento e imaginación

4. Disposición al aprendizaje

5. Relaciones sociales y redes sociales

6. Respeto, dignidad y reconocimiento

7. Integridad emocional, emociones

8. Integridad corporal

9. Empoderamiento

\section{Marco teórico}

3.1 El enfoque de las capacidades como marco teórico del desarrollo de competencias educativas.

La teoría del enfoque de las capacidades proporciona un marco normativo ideal para evaluar el alcance del bienestar individual y para desarrollar la naturaleza humana. La expresión capacidades, "capabilities", se refiere a potencialidades del ser humano, libertades sustantivas que disponen los individuos para desarrollar funcionamientos que les permitan realizarse y alcanzar el bienestar (Nussbaum, 2012:40). No obstante Sen y Nussbaum, difieren en algún aspecto de la teoría, en el sentido de que Sen prefiere el término funcionamiento al de capacidad. Define al funciona- 
miento -o funcionalidad- como las cosas que el individuo hace o la situación en la que se encuentra, debido a sus recursos y al uso que hace de ellos (Sen, 1987: 36).

El punto de partida es, por tanto, que el nivel o calidad de vida no se mide sólo por los bienes materiales sino, y principalmente, por los funcionamientos. Así, tanto Sen ${ }^{1}$ (2004) como Nussbaum (2002: 40) ${ }^{2}$ coinciden en que el nivel de vida y el bienestar alcanzado viene determinado por el grado de desarrollo de las capacidades, no por la cantidad de ingresos, ni por sus características ni por la utilidad que puedan generar sino por la oportunidad de transformar estos ingresos y recursos en funcionamientos valiosos. (Sen, 2004:15).

Martha C. Nussbaum elabora una lista de 10 capacidades que considera básicas para el desarrollo humano, defendiendo que todo país ha de garantizar, a través de sus políticas públicas, un umbral mínimo de desarrollo de estas 10 capacidades básicas (Nussbaum, 2012: 40-44). La mayoría de ellas, como veremos, se adquieren a través de la educación ${ }^{3}$ :

Vida. Vivir una vida de duración "normal". No morir de forma prematura o antes de que la vida se vea tan consumida que no valga la pena vivirla.

Salud física. Mantener una buena salud, también la salud reproductiva. Recibir una alimentación adecuada y disponer de un lugar adecuado para vivir.

Integridad Física. Estar protegido de cualquier ataque hacia la persona, poder vivir sin peligro. Poder desplazarse de un lugar a otro sin preocupación, estar protegidos de las agresiones sexuales o violencia domestica. Tener oportunidades de satisfacción sexual y elección en cuestiones reproductivas.

Sentidos, imaginación y pensamiento. Poder utilizarlos de un modo "verdaderamente humano". Tener alfabetización y formación matemática y científica básica. Usar la imaginación y el pensamiento para experimentar y producir obras o actos religiosos, musicales o parecidos, según se desee. Usar la mente en condiciones protegidas por las garantías de libertad de expresión política y artística, y por la libertad de práctica religiosa. Disfrutar de experiencias placenteras y evitar el dolor no beneficioso.

Emociones. Sentir emociones por cosas o personas externas a nosotros o por nosotros mismos. Amar a quienes nos aman y se preocupan por nosotros, sentir duelo por su ausencia. Poder amar, apenarse, sentir añoranza, gratitud e indignación justificada. Que no se malogre nuestro desarrollo emocional por culpa del miedo la ansiedad.

Razón Práctica. Reflexionar acerca de la planificación de la propia vida. Poder formarse una concepción del bien.

Afiliación.Poder vivir por y para los demás, disponer de las bases para no sentir humillación y si respeto por nosotros mismos. Ser capaces de imaginar la situación de otro u otra. Disponer de las bases sociales necesarias para no sentirnos humillados y sintamos respeto por nosotros mismo, que se nos trate con dignidad. Introducir 
disposiciones que combatan la discriminación por razón de raza, sexo, orientación sexual, etnia, casta, religión u origen nacional.

Otras especies. Vivir con los animales, plantas y entorno natural de una manera próxima y respetuosa

Juego. Disfrutar de actividades recreativas.

Control sobre el propio entorno. Político y material. Participar de forma efectiva en las decisiones políticas que gobiernan nuestra vida, tener derecho a la participación política y a la protección de la libertad de expresión y asociación. Poder poseer propiedades y derechos de propiedad en igualdad de condiciones con las demás personas, tener derecho a buscar trabajo en un plano de igualdad con los demás; estar protegidos legalmente frente a registros y detenciones que no cuenten con la debida autorización judicial. En el entorno laboral, ser capaces de trabajar como seres humanos, ejerciendo la razón práctica y manteniendo relaciones valiosas y positivas de reconocimiento mutuo con otros trabajadores y trabajadoras.

La educación juega un papel fundamental en el desarrollo de todas las capacidades humanas. La educación así entendida no se limita a aumentar los conocimientos sino que también desarrolla habilidades, actitudes y capacidades, "el analfabetismo es una discapacidad duradera”, concluye la filósofa. (Nussbaum, 2012: 181).

\subsection{Las competencias transversales del EEES y su relación con las capacidades}

Uno de los grandes retos del EEES ha sido la transición hacia modelos de aprendizaje que faciliten el desarrollo de las competencias. Esto supone superar un modelo educativo basado en el aprendizaje de conocimientos por otro fundamentado en desarrollo de habilidades, de "saber hacer". No es fácil dar una definición única de competencia, debido al carácter multidimensional del concepto que agrupa elementos actitudinales, comportamentales, contextuales y sociales (Colás, 2005)

Hay que decir que la mayoría de los autores convienen en que la competencia no es sólo un saber hacer, sino que convergen en ella el "saber" con el "saber hacer", pues no es algo meramente instrumental, hay que comprender e interiorizar por qué y para qué (Delors, 1996; Colás, 2005; ANECA, 2005; Zabala y Arnau, 2008,)

Las competencias posibilitan, fundamentalmente, que la persona que las desarrolla sea capaz de ejercer una actividad o profesión. En este sentido, el modelo de competencia universitaria corre el riesgo de centrar el aprendizaje universitario "en torno a productos formativos", a competencias profesionales y de cara al mercado laboral, en detrimento del desarrollo de otras competencias más sociales, emocionales, cívicas o morales. Creemos que el concepto de competencia debe ser indisociable del desarrollo integral de la persona, en la línea de la teoría de las capacidades anteriormente expuesta.

Si bien, no disponemos de un catálogo de capacidades educativas para el EEES. Hay algunos intentos de elaborar listas de capacidades que la educación superior 
debería desarrollar (Flores Crespo, 2002; Walker, 2006; Robeyns, 2003). Con ello se pretenden señalar las habilidades y oportunidades que deberían ser promovidas desde el EEES.

Las capacidades suponen una superación del concepto de competencia, unidad central de EEES y a la que algunos autores consideran demasiado instrumental. En este capítulo seguiremos la lista de capacidades propuesta por Melanie Walker (2006) para desarrollar en el EEES (Boni Aristizábal, 2010) a la que hemos añadido el empoderamiento, al considerar su desarrollo, una destreza fundamental parar formar parte de una sociedad constructiva. Y las relacionaremos con las competencias genéricas o transversales del EEES, definidas, como veremos, como las necesarias para desarrollar una vida personal y socialmente valiosos. Nuestra pretensión es la de conectar el concepto de competencias transversales con el de capacidades.

En realidad, el término competencia procede de la teoría lingüística de Chomsky (Feito Alonso, 2008). Está inspirado en la idea de que la escuela deber formar personas no sólo para que puedan participar en el mundo del trabajo sino también para que puedan desarrollar un proyecto personal de vida. Sin duda, la aproximación del currículum educativo y de la educación, en general, al concepto de competencia tiene como meta luchar contra la educación entendida sólo como transmisión del conocimiento y contra la fragmentación del mismo en asignaturas. La hipótesis subyacente es la de aislar un conjunto de competencias comunes a todos los sistemas educativos y aceptarlo como meta común, en un primer nivel, de la escolarización obligatoria por considerar dichas competencias necesarias para una vida digna.

La inspiración de este concepto de competencias en la teoría del enfoque de las capacidades es clara. En 1999 la OCDE, lanzó un programa de tres años de duración llamado: "Definition and Selectión of Competencies" (DeSeCo), con el objetivo de identificar un conjunto de competencias básicas para llevar vidas responsables y exitosas en una sociedad moderna y democrática (DeSeCo, 1999: 5). Una de los principales conclusiones que arrojaron los informes de la OCDE fue, precisamente, que la educación es algo más amplio que la adquisición de conocimientos y que la idea de competencia propuesta en dicho informe debería ir más allá del contexto escolar, apuntando a la necesidad de "preparar para la vida". (DeSeCo, 1999: 7).

En 2006 el Diario Oficial de la Unión Europea publicó el texto: "competencias clave para el aprendizaje permanente", en el que se especifican las competencias fundamentales a desarrollar en el ámbito escolar, y recomienda a los estados miembros la incorporación en sus currículum de las competencias clave (2006/962/CE). Posteriormente, se establecieron las competencias necesarias para el diseño de los grados y postgrados en el EEES. Dicho diseño fue realizado de acuerdo con la clasificación establecida en el Proyecto TUNING "The Tuning Educational Structures in Europa Project" (González y Wagenaar, 2003), y distingue entre:

Competencias generales o transversales: son aquellas que son transferibles y comunes a cualquier perfil profesional. 
Competencias específicas: son aquellas que son propias a cualquier perfil profesional por lo que otorgan identidad y consistencia a cualquier profesión.

Finalmente se estableció una última distinción, que separaba en varias áreas más las competencias transversales o generales. Con la intención de definir su especificidad, se clasificaron de la siguiente manera:

Competencias instrumentales: son aquellas que tienen un carácter de herramienta, una función instrumental. Suponen una combinación de habilidades manuales y capacidades cognitivas que posibilitan la competencia profesional.

Competencias interpersonales: suponen habilidades personales e interpersonales. Se refieren a la capacidad o habilidad que posibilita la colaboración en objetivos comunes. Estas destrezas implican capacidades de objetivación, planificación e información que favorecen procesos de cooperación e interacción social.

Competencias sistémicas: suponen destrezas y habilidades relacionadas con la totalidad de un sistema. Requieren una combinación de imaginación, sensibilidad y habilidad que permite ver cómo se relacionan y conjugan las partes de un todo. Requieren haber adquirido previamente las competencias instrumentales e interpersonales que constituyen la base de las competencias sistémicas.

Nuestra hipótesis es que las competencias transversales, comunes a todos los grados del EEES, deben recoger las capacidades básicas para el desarrollo humano preconizadas por la teoría del enfoque de las capacidades: razón práctica, emociones, sentidos, imaginación y pensamiento, afiliación y control sobre el propio entorno. Ello significaría que deberían ser el objetivo central en el desarrollo de la persona en su paso por la educación superior.

\section{Resultados y discusión}

En el cuadro $n^{\circ} 1$ se aprecia que el alumnado considera que se ha producido una adquisición bastante homogénea de las capacidades relacionadas con el conocimiento y con la inteligencia racional dado que todas ellas están valoradas en un nivel de adquisición que oscila entre el 6,84 y 6,9. Destaca, de entre todas las anteriores, conocimiento e imaginación al ser la que obtiene la puntuación más alta de entre ellas, al alcanzar casi un 7. Aunque también aprobada y muy cerca de las anteriores, la capacidad de empoderamiento se sitúa en un nivel inferior a las anteriores con un 6,46 .

El grado de obtención por los y las participantes de las capacidades integridad emocional, emociones e integridad corporal, de un 2,06 y un 2,31, respectivamente, resulta muy llamativo. 
Cuadro n¹. Puntuación de las capacidades básicas

\begin{tabular}{|l|c|}
\hline Capacidad & \\
\hline 1. Razón práctica & 6,85 \\
2. Resiliencia & 6,84 \\
\hline 3. Conocimiento e imaginación & 6,95 \\
\hline 4. Disposición al aprendizaje & 6,9 \\
\hline 5. Relaciones sociales y redes sociales & 6,84 \\
\hline 6. Respeto, dignidad y reconocimiento & 6,87 \\
\hline 7. Integridad emocional, emociones & 2,06 \\
\hline 8. Integridad corporal & 2,31 \\
\hline 9. Empoderamiento & 6,46 \\
\hline
\end{tabular}

M. Nussbaum (2002); M. Walker(2006). Elaboración propia.

Bajando un nivel en el análisis, el cuadro $n^{\circ} 2$ nos muestra cómo las competencias más valoradas en su adquisición son las interpersonales, en la mayoría de los supuestos, esto es, en la capacidad de resiliencia, conocimiento e imaginación, disposición al aprendizaje, relaciones sociales y redes, respeto, dignidad y reconocimiento e, incluso, son las únicas apreciadas en la capacidad de integridad corporal. De entre todas ellas, destaca la valoración dada a la interpersonal disposición al aprendizaje por ser la más elevada de todas con un 7,37.

Cuadro n². Relación competencias- capacidades. Puntuación medias ${ }^{4}$

\begin{tabular}{|c|c|c|}
\hline Capacidad & Competencias transversales & \\
\hline \multirow[t]{4}{*}{ 1.Razón práctica } & & 6,85 \\
\hline & a) Instrumentales & 6,64 \\
\hline & b) Interpersonales & 6,96 \\
\hline & c) Sistémicas & 6,97 \\
\hline \multirow[t]{4}{*}{ 2. Resiliencia } & & 6,84 \\
\hline & a) Instrumentales & 6,88 \\
\hline & b) Interpersonales & 6,9 \\
\hline & c) Sistémicas & 6,75 \\
\hline \multirow[t]{4}{*}{ 3. Conocimiento e imaginación } & & 6,95 \\
\hline & a) Instrumentales & 6,95 \\
\hline & b) Interpersonales & 7,01 \\
\hline & c) Sistémicas & 6,9 \\
\hline \multirow[t]{4}{*}{ 4. Disposición al aprendizaje } & & 6,9 \\
\hline & a) Instrumentales & 6,71 \\
\hline & b) Interpersonales & 7,37 \\
\hline & c) Sistémicas & 6,64 \\
\hline 5. Relaciones sociales y redes sociales & & 6,84 \\
\hline
\end{tabular}




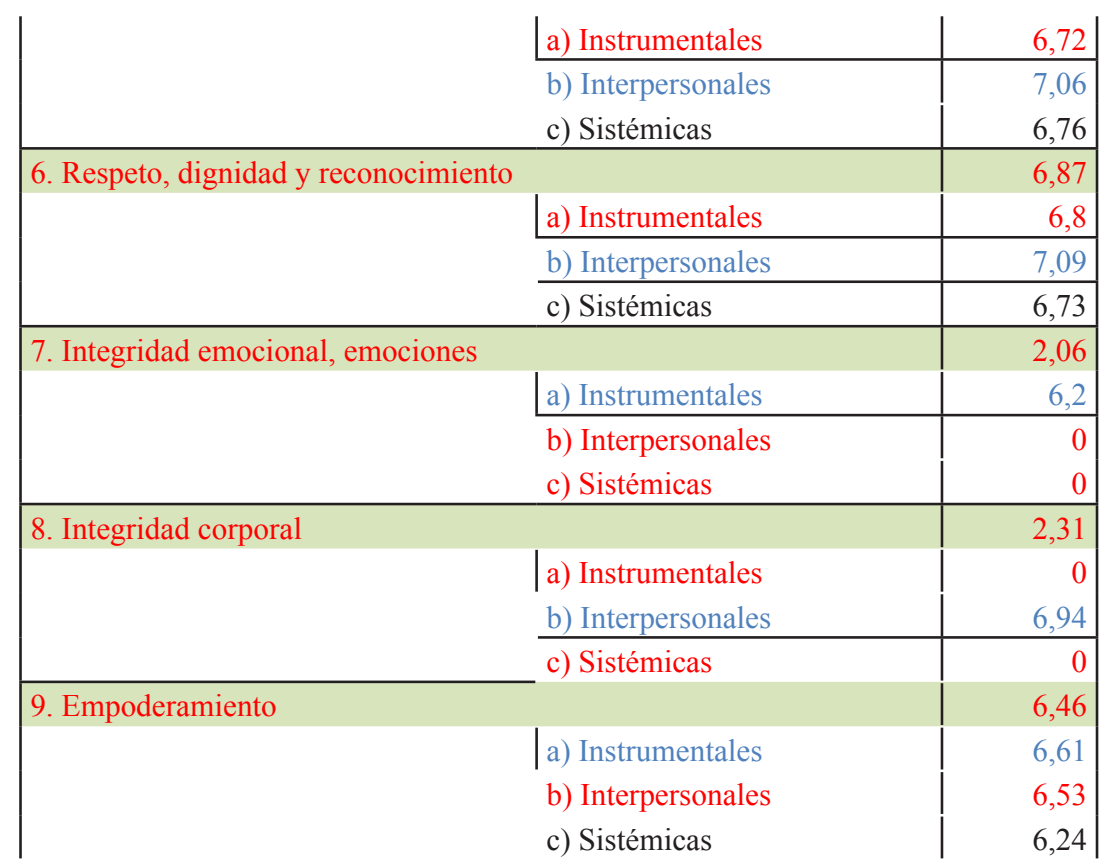

Fuente: M. Nussbaum (2002); M. Walker(2006). Elaboración propia.

El hecho es que son las competencias interpersonales las más valoradas en la mayoría de las capacidades. No obstante, si pasamos a describir cuales son las competencias concretas que conforman las interpersonales, podemos apreciar que las mismas no tienen como objetivo directo la adquisición de las capacidades emocionales, aún cuando muchas de ellas las presuponen. De otra parte, destaca que las competencias menos valoradas en su adquisición son las instrumentales en la mayoría de las capacidades.

Haciendo un tercer nivel de análisis, hemos desagregado los datos por sexos. Estos son los resultados de nuestro experimento:

Cuadro n⿳3 ${ }^{\mathbf{3}}$. Puntuación de las capacidades básicas por sexo

\begin{tabular}{|l|c|c|c|}
\hline \multicolumn{1}{|c|}{ Capacidad } & \multicolumn{1}{c|}{$\begin{array}{c}\text { Descrip } \\
\text { Media }\end{array}$} & $\begin{array}{c}\text { Sexo H } \\
\text { Media }\end{array}$ & $\begin{array}{c}\text { Sexo M } \\
\text { Media }\end{array}$ \\
\hline 1. Razón práctica & 6,83 & 6,40 & 7,22 \\
\hline 2. Resiliencia & 6,88 & 6,36 & 7,32 \\
\hline 3. Conocimiento e imaginación & 6,96 & 6,97 & 7,02 \\
\hline 4. Disposición al aprendizaje & 6,91 & 6,60 & 7,24 \\
\hline 5. Relaciones sociales y redes sociales & 6,86 & 6,44 & 7,25 \\
\hline 6. Respeto, dignidad y reconocimiento & 6,88 & 6,53 & 7,25 \\
\hline 7. Integridad emocional, emociones & 2,07 & 1,92 & 2,23 \\
\hline
\end{tabular}




\begin{tabular}{|l|c|c|c|}
\hline 8. Integridad corporal & 2,31 & 2,20 & 2,45 \\
\hline 9. Empoderamiento & 6,47 & 6,17 & 6,70 \\
\hline
\end{tabular}

Fuente: elaboración propia. Los dos valores más altos de cada columna

Los dos valores más bajos de cada columna

De este cuadro se extraen tres resultados. El primero y más relevante es que las alumnas ven incrementadas sus capacidades tras el paso por la universidad en mayor medida que los alumnos, lo que sucede con el conjunto de las capacidades, sin excepción alguna. El segundo viene a confirmar los resultados anteriormente extraídos de los cuadros $\mathrm{n}^{\circ} 1$ y n 2 : las capacidades relacionadas con la integridad, ya sea emocional ya lo sea corporal, son los menos desarrollados en ambos sexos, aún cuando en las mujeres lo son más que en los hombres.

Como tercer resultado, el cuadro nos indica que las capacidades que los alumnos consideran más desarrolladas tras su paso por la universidad son conocimiento e imaginación (3), y la disposición al aprendizaje (4), mientras que en las alumnas lo es resiliencia (2) y respeto, dignidad y reconocimiento(6).

\section{Conclusiones}

La práctica totalidad de competencias establecidas como necesarias en el EEES -competencias transversales- como necesarias para alcanzar por todo el alumnado universitario tienen su trasunto en las capacidades que permiten el bienestar de las personas.

Los resultados obtenidos en nuestro ejercicio, y en su visión más general, muestran que la práctica totalidad de las capacidades estudiadas están desarrolladas o incluidas en las competencias transversales, con las únicas excepciones de las capacidades relacionadas con la integridad, ya sea ésta la física o la emocional.

Esto es, los datos evidencian tres circunstancias relevantes. La primera de ellas es que la capacidad más desarrollada por los estudios universitarios es la razón práctica destacando, en este marco, el posicionamiento de la competencia responsabilidad.

La segunda, que llama poderosamente la atención, es que las capacidades integridad emocional, emociones e integridad corporal están completamente ausentes en las competencias transversales.

En tercer lugar, tras realizar la desagregación de los datos por sexos, resulta que el paso por los estudios universitarios de las alumnas incide más en el desarrollo de sus capacidades que en los hombres. Esto nos lleva a concluir que el diseño de las competencias tal y como está realizado contribuye al fomento de la igualdad de género en el desarrollo de las capacidades de estas. 
No obstante, los dos primeros resultados nos llevan a concluir que los estudios universitarios siguen teniendo como objetivo el desarrollo de la inteligencia racional pero no de la inteligencia emocional. En este sentido, el modelo de competencia universitaria corre el riesgo de centrar el aprendizaje universitario "en torno a productos formativos", a competencias profesionales y de cara al mercado laboral, en detrimento del desarrollo de otras competencias más sociales, emocionales, cívicas o morales. Creemos que el concepto de competencia debe ser indisociable del desarrollo integral de la persona, en la línea expuesta. Que el desarrollo de una vida personal y socialmente valiosa, pasa por un desarrollo completo de todas las capacidades.

\section{Bibliografía}

ANECA (2005). Libro Blanco del titulo de grado en pedagogía y educación social. Disponible en: http://www.aneca.es/var/media/150392/libroblanco_pedagogia1_0305.pdf. [30- 09-2013].

BECKER, G.S. (1993). Human capital: A theoretical and Empirical Analysis with special Reference to Education ( $3^{\mathrm{a}} \mathrm{ed}$.). Chicago: University of Chicago Press.

BONI ARISTIZÁBAL, A. (2010). "La educación superior desde el enfoque de capacidades. Una propuesta para el debate". REIFOP, 13 (3), 123-131. Disponible en: http://www.aufop.com/aufop/uploaded_files/articulos/1287011176.pdf. [30- 092013]

BRICEÑO MOSQUERA, A. (2011). "La educación y su efecto en la formación de capital humano y en el desarrollo económico de los países". En Apuntes de CENES, Vol. 30, no51, págs. 45-59. Disponible en: http://dialnet.unirioja.es/ servlet/articulo?codigo $=3724527$. [30- 09-2013]

COLÁS, P. (2005). "La formación universitaria en base a competencias". En P Colás y J. De Pablos. La universidad en la Unión Europea. El espacio Europeo de Educación Superior y su impacto en la docencia. Málaga: Aljibe.

DELORS, J. (1996). "La educación encierra un tesoro". Informe UNESCO de la Comisión Internacional sobre la Educación para el Siglo XXI. Disponible en: http://www.unesco.org/education/pdf/DELORS_S.PDF. [30- 09- 2013].

DIARIO OFICIAL DE LA UNIÓN EUROPEA (2006/ 962/CE). "Competencias clave para el aprendizaje permanente". Consultado en línea: http://eurlex.europa. eu/LexUriServ/LexUriServ.do?uri=OJ:L:2006:394:0010:0018:es:PDF. [30- 092013].

FEITO ALONSO, R. (2008). "Competencias educativas: hacia un aprendizaje genuino". En Andalucía Educativa $n^{\circ}$ 66. Abril 2008. Disponible en http://www. juntadeandalucia.es/averroes/ escuelatic20/didactica/Andalucia_educativa_ competencias_educativas.pdf. [30- 09- 2013].

FLORES CRESPOO, P. (2002). "En busca de nuevas explicaciones sobre la educación y la desigualdad. El caso de la Universidad Tecnológica de Nezahualcóyotl". Revista Mexicana de Investigación Educativa, 7: 17. México. Disponible en: http://www.redalyc.org/articulo.oa?id=14001606. [30- 09- 2013] 
FLORES-CRESPO, P. (2005). Educación superior y desarrollo humano. El caso de tres universidades tecnológicas. Biblioteca de la Educación Superior. Universidad Iberoamericana: México.

GONZÁLEZ, J. Y WAGENAAR, R. (2003): "Tuning Educational Structures in Europe". Informe Final. Disponible en:http://tuning.unideusto.org/tuningal/index. php?option $=$ com_docman\&Itemid $=191 \&$ task $=$ view_category\&catid $=22 \&$ order $=$ dmdate published $\&$ ascdesc $=$ DESC. [30- 09- 2013]

NUSSBAUM, M. C. (2002): Las mujeres y el desarrollo humano. El enfoque de las capacidades. Barcelona. Herder.

(2012): Crear capacidades. Propuesta para el desarrollo humano. Barcelona. Paidós.

OCDE, Organización para la Cooperación y el Desarrollo Económico (2005):“La definición y selección de competencias clave. Resumen ejecutivo". Disponible en línea:http://www.deseco.admin.ch/bfs/deseco/en/index/03/02.parsys. 78532. down. [30- 09- 2013]

OCDE. Organización para la Cooperación y el Desarrollo Económico (1999). DeSeCo. Proyectos sobre Competencias en el contexto de la OCDE. Análisis de base teórica y conceptual. Disponible en línea http://www.deseco.admin.ch/ bfs/deseco/en/index/03/02.parsys.78532.downloadList.94248.DownloadFile. tmp/2005.dscexecutivesummary.sp.pdf. [30- 09- 2013]

PÉREZ GÓMEZ, A. (1988). La cultura escolar en la sociedad neoliberal. Madrid: Morata.

ROBEYNS, I. (2003). "Sen's capability approach and gender inequality: select in grelevant capabilities" Feminist Economics, 9, pp.61-69. Routledge. Taylor \& Francis Group. Disponible en: http://csde.washington.edu/ scurran/files/readings/April28/recommended/SelectingRelevantCapabilities.pdf. [30- 09- 2013]

SCHULTZ, T. W. (1963). The Economic Value of Education. Nueva York: Columbia University Press.

SEN, Amartya K. (1987). Sobre ética y economía. Madrid: Alianza Editorial. (2004). Nuevo examen de la desigualdad. Madrid: Alianza Editorial.

SOLOW, M. (1956). "A Contribution to the Theory of Economic Growth", Quarterly Journal of Economics, LXX.

WALKER, M. (2006). Higher education Pedagogies. Berkshire: McGraw-Hill Education. Open University press,

ZABALA, A. Y ARNAU, L. (2007). 11 ideas clave. Cómo aprender y enseñar competencias. Barcelona: Graó.

\section{Notas}

1 Sen, en el marco de las conferencias Tanner en la Universidad de Standford, expuso por primera vez su concepción de las capacidades, en un discurso denominado “iIgualdad de qué?"Equality of What? (1979). 
2 "En lugar de preguntar acerca de la satisfacción de la gente o de los recursos que la gente está en condicione de manejar, nosotros preguntamos qué es lo que la gente es capaz de ser o de hacer. Sen ha insistido también en que es en este espacio de las capacidades donde mejor pueden plantearse las preguntas acera de la igualdad y de la desigualdad social." (Nussbaum, 2002: 40)

3 Hemos puesto en cursiva las consideradas capacidades propiamente educativas, según se expondrá más adelante.

4 En azul las capacidades/competencias mejor puntuadas y en rojo las peor valoradas.

\section{Las autoras}

Gloria Alarcón García es Master en Bussines Administration (1987), Doctora en Derecho (1994) y Master internacional en igualdad y género en el ámbito público y privado (2009). Es profesora Titular de Economía Aplicada en el Departamento de Hacienda y Economía del Sector Público de la Universidad de Murcia, investigadora principal del grupo de investigación de la Universidad de Murcia: "El Observatorio fiscal: análisis de las políticas públicas", E-071/2, y está en posesión de dos sexenios de investigación. Sus líneas principales de investigación son el fraude fiscal y la conciencia fiscal, la fiscalidad internacional, la tributación ambiental, el análisis de las políticas públicas desde la perspectiva de género, los gastos en infraestructuras desde la perspectiva de género, el presupuesto con perspectiva de género, el presupuesto participativo y la teoría del enfoque de las capacidades aplicada a las políticas públicas.

Cristina Guirao Mirón, catedrática de EE.MM. en excedencia desde 2011 y profesora asociada en las Universidades de Alicante (2003/2004) y Murcia (2004/2010). Desde 2011 contratada doctora en la Universidad de Murcia, departamento de Sociología y Trabajo Social. Doctora en Sociología por la Universidad de Alicante año 2010, La construcción social del problema de la conciliación vida familiar y laboral en España. Años 1999 a 2009. Autora de dos libros: Sociología (2000) y Curso de sociología (2007) y varios artículos: "Políticas públicas y espacio doméstico" (2009), "Modernidad y postmodernidad en el feminismo contemporáneo" (2010). Coordinadora de los cursos: "Los desafíos educativos de la Región de Murcia. Una educación para la igualdad, la ciudadanía y la corresponsabilidad" (Universidad del Mar, Murcia, 2009); "Los desafios educativos de España: los objetivos de Lisboa 2010" (Universidad del Mar, Murcia, 2010). 